\title{
Asymptotic behavior of solutions to the generalized Becker-Döring equations for general initial data
}

\author{
José Alfredo Cañizo Rincón \\ Dept. Matemática Aplicada \\ Universidad de Granada \\ E18071 Granada \\ Spain
}

10 July 2005

\begin{abstract}
We prove the following asymptotic behavior for solutions to the generalized Becker-Döring system for general initial data: under a detailed balance assumption and in situations where density is conserved in time, there is a critical density $\rho_{s}$ such that solutions with an initial density $\rho_{0} \leq \rho_{s}$ converge strongly to the equilibrium with density $\rho_{0}$, and solutions with initial density $\rho_{0}>\rho_{s}$ converge (in a weak sense) to the equilibrium with density $\rho_{s}$. This extends the previous knowledge that this behavior happens under more restrictive conditions on the initial data. The main tool is a new estimate on the tail of solutions with density below the critical density.
\end{abstract}

\section{Introduction}

Coagulation-fragmentation equations are useful as models that describe the dynamics of many physical phenomena in which a large number of particles or units can stick together to form groups of particles, or clusters. A first version of them was initially proposed by Becker and Döring [4], and a variant by Penrose and Lebowitz [19]; these relatively simple models take into account only processes in which a cluster gains or loses one particle, and describe only the concentration of clusters of a given size at a certain moment, omitting also a description of their spatial distribution. Since then a number of generalizations have been studied which also allow reactions between clusters of more than one particle, the main examples of this being the discrete coagulationfragmentation equations (see for example 3, 5, 6]), their continuous version [11, 17, 20, 21, 22, 9, 8, 18, and the respective versions including a spatial description by means of diffusion [12, 13]. A recent review can be found in [14]. 
The generalized Becker-Döring equations are an intermediate step between the Becker-Döring system and the full discrete coagulation-fragmentation equations in which we allow reactions between clusters of at most a given finite size $N$ and other clusters. The system of equations is the following:

$$
\begin{array}{llr}
\dot{c}_{1} & =-\sum_{k=1}^{\infty} W_{1, k} & \\
\dot{c}_{j} & =\frac{1}{2} \sum_{k=1}^{j-1} W_{j-k, k}-\sum_{k=1}^{\infty} W_{j, k}, & 2 \leq j \leq N \\
\dot{c}_{j}=\frac{1}{2} \sum_{k=1}^{j-1} W_{j-k, k}-\sum_{k=1}^{N} W_{j, k}, & N+1 \leq j \leq 2 N \\
\dot{c}_{j}=\sum_{k=1}^{N} W_{j-k, k}-\sum_{k=1}^{N} W_{j, k}, & j \geq 2 N+1
\end{array}
$$

Here the unknowns are $c_{j}=c_{j}(t)$ for $j=1, \ldots$, positive functions depending on the time $t$ which are intended to represent the density of clusters of size $j$ (those formed by $j$ elementary particles). The quantities $W_{j k}$, which depend on the $c_{j}$, are given by

$$
W_{j k}:=a_{j k} c_{j} c_{k}-b_{j k} c_{j+k} \quad(j, k \geq 1),
$$

where the numbers $a_{j k}, b_{j k}$ for $j, k \geq 1$ with $\min \{j, k\} \leq N$ are the coagulation and fragmentation coefficients, respectively, which are symmetric in $j, k$. As can be seen, this system is a particular case of the coagulation-fragmentation equations when $a_{j k}=b_{j k}=0$ if $\min \{j, k\}>N$.

The study of the long-time behavior of solutions to these equations is expected to be a model of physical processes such as phase transition. Call $\sum_{j=1}^{\infty} j c_{j}$ the density of a solution $\left\{c_{j}\right\}_{j \geq 1}$. For the Becker-Döring equations it was proved in [1] and [2] that, under certain general conditions which include a detailed balance (see below), there is a critical density $\rho_{s} \in[0, \infty]$ such that any solution that initially has density $\rho_{0} \leq \rho_{s}\left(\rho_{0}<\infty\right.$ if $\left.\rho_{s}=\infty\right)$ will converge for large times, in a certain strong sense, to an equilibrium solution with density $\rho_{0}$, while any solution with density above $\rho_{s}$ will converge (in a weak sense) to the only equilibrium with density $\rho_{s}$. The rate of convergence to equilibrium was studied in [10]. The mentioned weak convergence can then be interpreted as a phase transition in the physical process modelled by the equation (see below for a precise statement). It is an interesting problem to extend this result to more general models; this has been done for the generalized Becker-Döring equations in [6] under some conditions on the decay of the initial data and in [7 for suitably small initial data. The aim of this paper is to prove that this result about the generalized Becker-Döring system is true for general initial data. The corresponding result is expected to hold for the full coagulation-fragmentation equations, but finding a proof of this is still an open problem. 
Acknowledgements. These results were obtained under the supervision and help of Stéphane Mischler. I wish to thank him for his explanations and suggestions. I would also like to thank the anonymous referees for their valuable corrections and comments. The author was supported by FPU grant AP2001-3940 and by EU financed network no. HPRN-CT-2002-00282.

\section{Statement of the main result}

Let us recall some usual definitions and notation from previous works on the coagulation-fragmentation equations. We will make use of the vector space

$$
X:=\left\{\left\{c_{j}\right\}_{j \geq 1}\left|\sum_{j=1}^{\infty} j\right| c_{j} \mid<\infty\right\}
$$

with norm

$$
\|c\|:=\sum_{j=1}^{\infty} j\left|c_{j}\right| \quad \forall c=\left\{c_{j}\right\}_{j \geq 1} \in X .
$$

The space $X$ is clearly a Banach space (actually, this space is isometric to the space of absolutely summable sequences under the map $\left.\left\{c_{j}\right\} \mapsto\left\{j c_{j}\right\}\right)$. In it we will make use of the notion of convergence associated to the norm $\|\cdot\|$, which we will call "strong convergence" following common usage. We will also say that a sequence $\left\{c^{i}\right\}_{i \geq 1}$ of elements of $X$ converges weak-* to an element $c \in X$, and will denote it by $c^{i} \stackrel{*}{\rightarrow} c$, if

1. there exists $M \geq 0$ such that $\left\|c^{i}\right\| \leq M$ for all $i \geq 1$ and

2. $c_{j}^{i} \rightarrow c_{j}$ when $i \rightarrow \infty$, for all $j \geq 1$ (where $c^{i}=\left\{c_{j}^{i}\right\}_{j \geq 1}$ and $c=\left\{c_{j}\right\}_{j \geq 1}$ ).

This is just the usual weak-* convergence in the space $X$ when it is regarded as the dual space of the space of sequences $\left\{c_{k}\right\}_{k \geq 1}$ such that $\lim _{k \rightarrow \infty} k^{-1} c_{k}=0$, with norm given by $\left\|\left\{c_{k}\right\}\right\|:=\max \left\{k^{-1}\left|c_{k}\right| \mid k \geq 1\right\}$ (see 11, p. 672). We also cite a result from [1]:

Lemma 1 ([1], Lemma 3.3). If $\left\{c^{n}\right\}$ is a sequence in $X$ such that $c^{n} \stackrel{*}{\rightarrow} c \in$ $X$ and $\left\|c^{n}\right\| \rightarrow\|c\|$, then $c^{n} \rightarrow c$ strongly in $X$.

The subset of $X$ formed by the sequences of nonnegative terms will be referred to as $X^{+}$:

$$
X^{+}:=\left\{\left\{c_{j}\right\}_{j \geq 1} \in X \mid c_{j} \geq 0 \forall j \geq 1\right\} .
$$

We will ask for any solution $\left\{c_{j}(t)\right\}_{j \geq 1}$ to be, for each fixed time $t$, in $X^{+}$; this is natural, given that densities should be positive and that the sum $\sum_{j=1}^{\infty} j c_{j}(t)$ represents the total density of particles at time $t$ (or total mass, depending on the interpretation given to the $c_{j}$ 's). More precisely, we will use the following concept of solution from [3], section 2: 
Definition 2. A solution on the interval $[0, T[$ (for a given $T>0$ or $T=\infty$ ) of (11) is a function $c:\left[0, T\left[\rightarrow X^{+}\right.\right.$such that, if we put $c(t)=\left\{c_{j}(t)\right\}_{j \geq 1}$ for $t \in[0, T[$,

1. $c_{j}:[0, T[\rightarrow \mathbb{R}$ is absolutely continuous for all $j \geq 1$ and $\|c(t)\|$ is bounded on $[0, T[$,

2. for all $j=1,2, \ldots$, the sums $\sum_{k=1}^{\infty} a_{j, k} c_{k}(t)$ and $\sum_{k=1}^{\infty} b_{j, k} c_{j+k}(t)$ are finite for almost all $t \in[0, T[$,

3. and equations (1) hold for almost all $t \in[0, T[$.

Remark 1. For convenience, this definition has been slightly changed with respect to that in [3]: it has been stated for the generalized Becker-Döring system instead of the full coagulation equations, and conditions have been phrased in different terms, but it can easily be checked that if the coefficients $a_{j k}, b_{j k}$ satisfy hypothesis 1 below then this concept of solution is equivalent to that in [3]. Hence, results from [3] are also applicable in our case, a fact that we will use later.

As we do not know of a uniqueness result that can be applied under the above hypotheses we need to define a concept of admissibility to precise which solutions our result applies to. In [6] this is done by choosing solutions which are limits of solutions to the finite set of equations obtained by truncating system (11). We will call these solutions Carr-da Costa admissible. Here we will define a slight modification of this concept: an admissible solution will be one which is the limit of Carr-da Costa admissible solutions with truncated initial data. The concept must of course be the same under any set of conditions that ensure uniqueness, but we have not found a sufficiently general uniqueness result and thus the following will be needed:

Definition 3. Take $T>0$ or $T=+\infty$. An admissible solution of the generalized Becker-Döring equations (11) on $\left[0, T\left[\right.\right.$ with initial data $c^{0}=\left\{c_{j}^{0}\right\}_{j \geq 1} \in X^{+}$ is a solution $c$ which is a limit in $L_{\text {loc }}^{\infty}([0, T[, X)$ of Carr-da Costa admissible solutions $c_{n}=\left\{c_{n}^{j}\right\}_{j \geq 1}$ of (1) with truncated initial data $c^{0, n}$ given by

$$
\begin{aligned}
& c_{j}^{0, n}:=c_{j}^{0} \text { for } j \leq n \\
& c_{j}^{0, n}:=0 \text { for } j>n .
\end{aligned}
$$

Remark 2. The above convergence is uniform in compact subsets of $[0, T[$, in the sense of the norm $\|\cdot\|$ in $X$; in particular, the functions $c_{j}^{n}$ in the definition converge uniformly when $n \rightarrow \infty$ in compact subsets of $\left[0, T\right.$ [ to $c_{j}$.

Below we state the conditions on the coefficients under which we will prove our result. Though in the equations only the coefficients $a_{j k}, b_{j k}$ with $\min \{j, k\} \leq N$ appear, for convenience we will use coefficients $a_{j k}, b_{j k}$ defined for all $j, k \geq 1$ and simply set $a_{j k}=b_{j k}=0$ if $\min \{j, k\}>N$. Thus we have hypothesis 1

Hypothesis 1 (Generalized Becker-Döring). There exists an $N \geq 2$ such that $a_{j k}=b_{j k}=0$ if $\min \{j, k\}>N$, and $a_{j k}, b_{j k}>0$ otherwise. 
Detailed balance is a physical assumption also used, for example, in [1, 6, which expresses the principle of microscopic reversibility from chemical kinetics; essentially, it states that equilibria of a certain form exist (see theorem 8 below):

Hypothesis 2 (Detailed Balance). There exists a positive sequence $\left\{Q_{j}\right\}_{j \geq 1}$ with $Q_{1}=1$ such that for all $j, k \geq 1$,

$$
a_{j k} Q_{j} Q_{k}=b_{j k} Q_{j+k}
$$

A certain bound on the growth rate of coefficients is known to be necessary to ensure the existence of density-conserving solutions [3, 8] (in other situations density is only conserved for a finite time after which density decreases, a phenomenon known as gelation); for our main result to be true (theorem 9) it is evidently necessary that density is conserved, so we impose a condition ensuring this.

Hypothesis 3 (Growth of coefficients). For some constants $K>0$ and $0 \leq \alpha<1$,

$$
\begin{aligned}
& a_{j k} \leq K\left(j^{\alpha}+k^{\alpha}\right), \\
& b_{j k} \leq K\left(j^{\alpha}+k^{\alpha}\right) .
\end{aligned}
$$

In the next hypothesis, (3) is a physical condition that asserts that any cluster has a lower free energy than its pieces taken separately (see [6], Remark 5.1); (4) will be seen to imply the existence of a critical density $\rho_{s}$ (the relationship between the following $z_{s}$ and this critical density is given below in 8):

Hypothesis 4. The sequence $Q_{j}$ satisfies:

$$
\begin{aligned}
& \log Q_{j}+\log Q_{k} \leq \log Q_{j+k} \quad \text { for all } j, k \geq 1, \\
& 0<\lim _{j \rightarrow \infty} \frac{Q_{j}}{Q_{j+1}}:=z_{s}<\infty .
\end{aligned}
$$

Remark 3. This implies that $\lim _{j \rightarrow \infty} \frac{Q_{j}}{Q_{j+m}}=z_{s}^{m}$ for $m \geq 1$ and that $\lim _{j \rightarrow \infty} Q_{j}^{1 / j}=$ $\frac{1}{z_{s}}$.

We also need to assume, as new hypotheses, a certain regularity of the coefficients:

Hypothesis 5. For $j, m=1, \ldots, N$,

$$
\frac{a_{j k}}{a_{j, k+m}} \rightarrow 1 \quad \text { when } k \rightarrow \infty
$$

Hypothesis 6. For some constant $K_{a}, j, m=1, \ldots, N$ and $k \geq 1$,

$$
\left|a_{j k}-a_{j, k+m}\right| \leq K_{a}
$$


Observe that hypotheses 5 and 6 are independent; for example, for $j=$ $1, \ldots, N$ and $k \geq 1, a_{j k}=\exp (-j-k)$ satisfies the second one but not the first; and $a_{j k}=[\log (j+k)] \sqrt{j+k}$ (with $[x]$ being the integer part of $x$ ) satisfies the first but not the second.

Remark 4 . The kind of coefficients allowed by the previous hypotheses are, for example, $a_{j k} \leq C\left(j^{\alpha}+k^{\alpha}\right)$ for $j=1, \ldots, N$ and $k \geq 1$, sufficiently regular to fulfill hypotheses 5 and 6 , and $b_{j k}$ given by hypothesis 2 with any choice of $Q_{j}$ satisfying (3) and (4). Note that (3) implies that $b_{j k} \leq a_{j k}$, so $b_{j k} \leq C\left(j^{\alpha}+k^{\alpha}\right)$ also. For a concrete example, pick $C_{1}, C_{2}>0$ and $\alpha, \delta \in[0,1[$ and define the following coefficients for $\min \{j, k\} \leq N$ :

$$
\begin{aligned}
a_{j k} & :=C_{1}\left(j^{\alpha}+k^{\alpha}\right) \\
b_{j k} & :=C_{1}\left(j^{\alpha}+k^{\alpha}\right) \exp \left(C_{2}\left((j+k)^{\delta}-j^{\delta}-k^{\delta}\right)\right) .
\end{aligned}
$$

The coefficients are taken to be zero when $\min \{j, k\}>N$. These correspond to $Q_{j}=\exp \left(C_{2}\left(j-j^{\delta}\right)\right)$ and have $z_{s}=e^{-C_{2}}$.

We borrow known existence results for the kind of admissible solutions of definition 3 from [3]:

Theorem 4 ([3], Theorems 2.4, 3.6 and 5.4). Assume hypotheses 1 and 3. and take $c^{0} \in X^{+}$. Then there exists an admissible solution $c$ to (1) on $\left[0,+\infty\left[\right.\right.$ with $c(0)=c^{0}$. Furthermore, under hypothesis 1] all solutions to (1) are density-conserving.

Remark 5. Theorem 2.4 in 3 gives the existence of a solution (in fact, a Carr-da Costa admissible solution by the method of construction). Theorem 3.6 from 3 . proves this solution conserves density. Finally, Theorem 5.4 in the same paper gives the existence of a solution that can be obtained as the uniform limit in compact sets of [0,T] of Carr-da Costa admissible solutions with truncated initial data, thus giving the existence of an admissible solution in the sense used here.

Lemma 5. Assume hypotheses 1] and [3. Take $\mu>1$ and suppose that $c=$ $\left\{c_{j}\right\}_{j \geq 1}$ is an admissible solution to (11) on $[0, T[$ for some $T>0$ with initial data $c(0)=c^{0}$ such that $\sum_{j=1}^{\infty} j^{\mu} c_{j}^{0}<+\infty$. Then $\sum_{j=1}^{\infty} j^{\mu} c_{j}(t)$ is finite for all $0 \leq t<T$.

Proof. This is just Theorem 3.3 in [6], stated for admissible solutions in the sense we use here. As Carr-da Costa admissible solutions satisfy the estimate given in the proof of the above theorem in [6] (which depends only on $\sum_{j=1}^{\infty} j^{\mu} c_{j}^{0}$ ), we can pass to the limit and thus prove that our admissible solutions also satisfy it.

Hypotheses 14 4 imply those of Theorems 5.1 and 5.2 in 6]: (1.7) and (H2) in 6] are always fulfilled if we assume hypothesis [1 (H1) is our 3 and (H3), (H4) from [6] are contained in hypotheses 1 and 4 here, respectively. This enables us to use these theorems here (recall remark 1); we will need the following one about the equilibrium solutions of (10). 
Definition 6. An equilibrium of (11) is a solution of (1) that does not depend on time. The density of an equilibrium $c$ is the norm of $c$ in $X, \sum_{j=1}^{\infty} j c_{j}$.

Definition 7. The critical density $\rho_{s}$ is defined to be

$$
\rho_{s}:=\sum_{j=1}^{\infty} Q_{j} z_{s}^{j}, \quad\left(0<\rho_{s} \leq \infty\right) .
$$

Theorem 8 ([6], Theorem 5.2). Assume hypotheses 1 \& 4

1. For $0 \leq \rho \leq \rho_{s}$ (and also $\rho<+\infty$ if $\rho_{s}=+\infty$ ), there exists exactly one equilibrium $\left\{c_{j}^{\rho}\right\}$ of (1) with density $\rho$, which is given by

$$
c_{j}^{\rho}=Q_{j} z^{j} \quad \forall j \geq 1
$$

where $z$ is the only positive number such that $\sum_{j=1}^{\infty} j Q_{j} z^{j}=\rho$.

2. For $\rho_{s}<\rho<+\infty$ there is no equilibrium of (11) with density $\rho$.

Observe that when $\rho_{s}$ is finite and $\left\{c_{j}^{\rho_{s}}\right\}_{j \geq 1}$ represents the critical equilibrium (the one with density $\rho_{s}$ ), $z_{s}$ is the single particle density $c_{1}^{\rho_{s}}$ of this equilibrium.

The main result in this paper is the following:

Theorem 9. Assume hypotheses 1, 6, and let $c=\left\{c_{j}\right\}_{j \geq 1}$ be an admissible solution of the generalized Becker-Döring equations (11) (whose existence is given by theorem 4). Call $\rho_{0}:=\sum_{j=1}^{\infty} j c_{j}(0)$, the initial density.

1. If $0 \leq \rho_{0} \leq \rho_{s}$ then $c$ converges strongly in $X$ to the equilibrium with density $\rho_{0}$.

2. If $\rho_{s}<\rho_{0}$ then $c$ converges in the weak-* topology to the equilibrium with density $\rho_{s}$.

\section{Proofs}

The following result from [6] already gives part of Theorem 9] Again, note that the hypotheses in 6 are contained in those here:

Theorem 10 ([6], Theorem 6.1). Assume hypotheses 1] 4. Let $c=\left\{c_{j}\right\}$ be a solution of (1) on $\left[0, \infty\left[\right.\right.$, and call $\rho_{0}:=\sum_{j=1}^{\infty} j c_{j}$.

Then there exists $0 \leq \rho \leq \min \left\{\rho_{0}, \rho_{s}\right\}$ such that $c \stackrel{*}{\rightarrow} c^{\rho}$, where $c^{\rho}$ is the only equilibrium of (1) with density $\rho$ (given by Theorem [8).

With Theorem [10 the next result will be enough to complete a proof of Theorem 9

Theorem 11. Assume hypotheses [1] 6] hold. Suppose that $c$ is an admissible solution to the generalized Becker-Döring equations (11) with initial data $c^{0} \in$ $X_{+}$such that c converges weak-* to an equilibrium with density $\rho<\rho_{s}$. Then, $c$ converges strongly to this equilibrium (and in particular, $\rho$ is the density of the solution $c$, i.e. $\left.\rho=\rho_{0}\right)$. 
Hence, the aim of the rest of this section will be to prove Theorem 11. The following key result gives a bound on the solutions that will easily imply the precompactness of the orbits, which in turn implies Theorem 11. Call, for $i \geq 1$,

$$
G_{i}(t) \equiv \sum_{j=i}^{\infty} j c_{j}(t) .
$$

Proposition 12. Let $c=\left\{c_{j}\right\}_{j \geq 1}$ be an admissible solution of the generalized Becker-Döring equations (11). Assume hypotheses [1,6.

Suppose that for some $z<z_{s}$

$$
c_{j}(t) \leq z_{j}:=z^{j} Q_{j} \quad \text { for all } j=1, \ldots, N \text { and all } t \geq 0 .
$$

Suppose that $\left\{r_{i}\right\}_{i \geq 1}$ is a strictly decreasing sequence of positive numbers that satisfy, for some $\lambda$ with $1<\lambda<z_{s} / z$ :

$$
\frac{r_{k-1}-r_{k}}{r_{k}-r_{k+1}}<\lambda \quad \text { for all } k
$$

and such that $G_{i}(0) \leq r_{i}$ for all $i$.

Then there exist a positive integer $k_{0}$ and a constant $C>0$ such that $G_{i}(t) \leq C r_{i}$ for all $i \geq k_{0}$ and all positive times.

The proof of proposition 12, which contains the core of the argument, is a generalization of a method used in unpublished notes by $\mathrm{Ph}$. Laurençot and $\mathrm{S}$. Mischler 15. This method is inspired by the proof of uniqueness of solutions to the Becker-Döring equation in [16]. The use of this kind of argument can be traced back to 2 .

Note that the condition on $\left\{r_{k}\right\}$ in proposition 12 is not very stringent as the following lemma states:

Lemma 13. Given $\lambda>1$ and a positive sequence $\left\{g_{k}\right\}_{k \geq 1}$ which tends to zero as $k$ tends to infinity, there exists a strictly decreasing positive sequence $\left\{r_{k}\right\}_{k \geq 1}$ which converges to zero, such that $g_{k} \leq r_{k}$ and

$$
\frac{r_{k-1}-r_{k}}{r_{k}-r_{k+1}} \leq \lambda \quad \text { for all } k
$$

Proof. Define

$$
\begin{aligned}
& \bar{g}_{1}:=\sup _{j \geq 1}\left\{g_{j}\right\}+1 \\
& \bar{g}_{k}:=\sup _{j \geq k}\left\{g_{j}\right\}, \quad \text { for } k \geq 2 \\
& h_{k}:=\bar{g}_{k}-\bar{g}_{k+1}, \quad \text { for } k \geq 1 .
\end{aligned}
$$

Then $\bar{g}_{k}$ is decreasing, tends to zero and for all $k$ we have $\bar{g}_{k}=\sum_{j=k}^{\infty} h_{j}$. Define $s_{k}$ recursively as:

$$
\begin{aligned}
s_{1} & :=h_{1} \\
s_{k+1} & :=\max \left\{\frac{s_{k}}{\lambda}, h_{k+1}\right\} .
\end{aligned}
$$


Then $s_{k}>0$ for all $k$ (it is to ensure this that we added 1 to $\bar{g}_{1}$ ) and we can see that $\sum_{k \geq 1} s_{k}$ converges. For this, note that $s_{k+1} \leq\left(s_{k} / \lambda\right)+h_{k+1}$ and write for $m \geq 2$ :

$$
\sum_{k=1}^{m+1} s_{k}=s_{1}+\sum_{k=1}^{m} s_{k+1} \leq h_{1}+\sum_{k=1}^{m} h_{k+1}+\frac{1}{\lambda} \sum_{k=1}^{m} s_{k} \leq \bar{g}_{1}-\bar{g}_{m+2}+\frac{1}{\lambda} \sum_{k=1}^{m} s_{k},
$$

so we have that

$$
\left(1-\frac{1}{\lambda}\right) \sum_{k=1}^{m} s_{k} \leq \bar{g}_{1},
$$

which proves the summability of $\left\{s_{k}\right\}$ since $\lambda>1$. (I thank the referees for suggesting a simpler version of this proof).

Clearly, $s_{k} \geq h_{k}$. Let us finally define

$$
r_{k}:=\sum_{j=k}^{\infty} s_{j} \geq \sum_{j=k}^{\infty} h_{j}=\bar{g}_{k} \geq g_{k}
$$

which is positive, greater than $g_{k}$, strictly decreasing, tends to zero as $k \rightarrow \infty$ and

$$
\frac{r_{k-1}-r_{k}}{r_{k}-r_{k+1}}=\frac{s_{k-1}}{s_{k}} \leq \lambda
$$

\subsection{Proof of the proposition}

We will prove the proposition for solutions whose initial data is a truncation at a sufficiently large finite size of $\left\{c_{i}(0)\right\}_{i \geq 1}$, with constants $C$ and $k_{0}$ that do not depend on the size of this truncation; then the proposition follows for general initial data by a standard approximation argument using definition 3 of an admissible solution.

Take an $L \geq 1$ and consider a solution $\left\{c_{i}^{L}\right\}_{i \geq 1}$ with initial data $c_{i}^{L}(0)=$ $c_{i}(0)$ for $i=1, \ldots, L$ and $c_{i}^{L}(0)=0$ for $i>L$. It is again enough to prove the bound in the result up to a finite time $T>0$, with a constant that does not depend on $T$. So fix $T>0$, and let us find $C$ and $k_{0}$ (independent of $L$ and T) such that

$$
c_{i}^{L}(t) \leq C r_{i} \quad \text { for all } i \geq k_{0}, \quad t \in[0, T] \text { and } L \text { sufficiently large. }
$$

By the admissibility of $c$ we know that the functions $c_{j}^{L}$ converge uniformly in $[0, T]$ to $c_{j}$ as $L \rightarrow \infty$ (see remark 2), so the hypotheses of the proposition imply that for sufficiently large $L$

$$
c_{j}^{L}(t)<z^{j} Q_{j} \text { for } j=1, \ldots, N, t \in[0, T] .
$$

In the following $L$ will always be large enough for this to hold (note that the choice of $L$ depends also on $T$ ). 
Furthermore, $G_{i}^{L}(0) \leq G_{i}(0) \leq r_{i}$ for all $i$ (where we have denoted $G_{i}^{L}=$ $\sum_{j=i}^{\infty} j c_{j}^{L}$, the corresponding to $G_{i}$ for the solution $\left.\left\{c_{i}^{L}\right\}\right)$.

From now, to simplify the notation a bit, we will omit the $L$ in both $c_{j}^{L}$ and $G_{j}^{L}$, as the full $c_{j}$ and $G_{j}$ will not be mentioned anymore. $W_{j k}$ will be used to denote $a_{j k} c_{j}^{L} c_{k}^{L}-b_{j k} c_{j+k}^{L}$.

For any sequence $\left\{\Psi_{j}\right\}_{j \geq 1}$ it holds formally that:

$$
\frac{d}{d t} \sum_{j=1}^{\infty} \Psi_{j} c_{j}=\frac{1}{2} \sum_{j, k=1}^{\infty}\left(\Psi_{j+k}-\Psi_{j}-\Psi_{k}\right) W_{j k}
$$

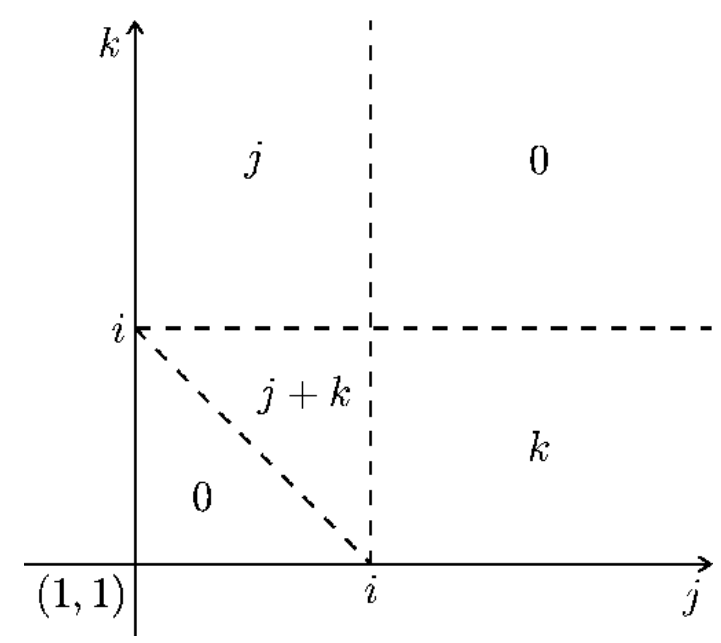

Figure 1: Values of $\Psi_{j+k}-\Psi_{j}-\Psi_{k}$

In particular we can apply the previous relation to $\Psi_{j}=j \cdot 1_{j \geq i}(i \geq 1)$ to get:

$$
\frac{d}{d t} \sum_{j=i}^{\infty} j c_{j}=\frac{1}{2} \sum_{j=1}^{i-1} \sum_{k=i-j}^{i-1}(j+k) W_{j k}+\sum_{j=1}^{i-1} \sum_{k=i}^{\infty} j W_{j k},
$$

and this equality is rigorously justified because the solution $\left\{c_{i}\right\}$ has finite moments $\sum_{j=1}^{\infty} j^{\mu} c_{j}^{L}(t)$ of every order $\mu \in \mathbb{R}$ for every positive time $t$ (see lemma 5), so the sums on both sides of the previous equality converge uniformly and we can obtain the equation by means of standard results on differentiation of uniformly convergent series of functions. One way to obtain the expression on the right hand side is to write the sum over $j, k$ as a sum over the regions depicted in figure 1 where the value of $\Psi_{j+k}-\Psi_{j}-\Psi_{k}$ is indicated in each of them.

Due to hypothesis 1. $W_{j k}=0$ if $\min \{j, k\}>N$. Hence, for $i>2 N$ the first sum in (5) (which comprises all pairs $j, k<i$ such that $j+k \geq i$ ) can be 
broken into those terms where $j \leq N$ and those where $k \leq N$ :

$$
\begin{array}{r}
\frac{1}{2} \sum_{j=1}^{i-1} \sum_{k=i-j}^{i-1}(j+k) W_{j k}=\frac{1}{2} \sum_{j=1}^{N} \sum_{k=i-j}^{i-1}(j+k) W_{j k}+\frac{1}{2} \sum_{j=i-N}^{i-1} \sum_{k=i-j}^{i-1}(j+k) W_{j k} \\
=\frac{1}{2} \sum_{j=1}^{N} \sum_{k=i-j}^{i-1}(j+k) W_{j k}+\frac{1}{2} \sum_{k=1}^{N} \sum_{j=i-k}^{i-1}(j+k) W_{j k} \\
=\sum_{j=1}^{N} \sum_{k=i-j}^{i-1}(j+k) W_{j k}
\end{array}
$$

where we have changed the order of the double sum and used the symmetry of $(j+k) W_{j k}$. If $i>N$, the second sum in (5) is nonzero only if $j \leq N$, so for $i>2 N$ we have

$$
\frac{d}{d t} \sum_{j=i}^{\infty} j c_{j}=\sum_{j=1}^{N} \sum_{k=i-j}^{i-1}(j+k) W_{j k}+\sum_{j=1}^{N} \sum_{k=i}^{\infty} j W_{j k} .
$$

We rewrite the latter double sum:

$$
\begin{aligned}
& \sum_{j=1}^{N} \sum_{k=i}^{\infty} j W_{j k}=\sum_{j=1}^{N} \sum_{k=i}^{\infty} j\left(a_{j k} c_{j} c_{k}-b_{j k} c_{j+k}\right) \\
&= \sum_{j=1}^{N} \sum_{k=i-j}^{\infty} j a_{j, j+k} c_{j} c_{j+k}-\sum_{j=1}^{N} \sum_{k=i}^{\infty} j b_{j k} c_{j+k} \\
&=\sum_{j=1}^{N} \sum_{k=i-j}^{i-1} j a_{j, j+k} c_{j} c_{j+k}+\sum_{j=1}^{N} \sum_{k=i}^{\infty} j c_{j+k}\left(a_{j, j+k} c_{j}-b_{j k}\right) \\
&=: S_{1}+S_{2}
\end{aligned}
$$

where we have denoted the two double sums as $S_{1}, S_{2}$ to mention them later.

We know that

$$
c_{j}(t) \leq Q_{j} z^{j} \quad \text { for all } j=1, \ldots, N \text { and } t \in[0, T] .
$$

Hence, as $z<z_{s}$, we see that thanks to hypothesis 2 and for $j \in\{1, \ldots, N\}$,

$$
\begin{aligned}
a_{j, j+k} c_{j}-b_{j k} \leq a_{j, j+k} Q_{j} z^{j}-a_{j k} Q_{j} \frac{Q_{k}}{Q_{j+k}} & \\
& =Q_{j} a_{j, j+k}\left(z^{j}-\frac{a_{j k}}{a_{j, j+k}} \frac{Q_{k}}{Q_{j+k}}\right) .
\end{aligned}
$$

Note that the term in parenthesis tends to $z^{j}-z_{s}^{j}$ as $k \rightarrow \infty$ (thanks to hypotheses 5 and (4), so $S_{2} \leq 0$ for $t \in[0, T]$ and $i$ sufficiently large. Then, continuing from (6), using (7) and omitting $S_{2}$, we have for $i$ large that

$$
\frac{d}{d t} \sum_{j=i}^{\infty} j c_{j} \leq \sum_{j=1}^{N} \sum_{k=i-j}^{i-1}(j+k) W_{j k}+\sum_{j=1}^{N} \sum_{k=i-j}^{i-1} j a_{j, j+k} c_{j} c_{j+k}
$$


Using again $c_{j} \leq z_{j}:=z^{j} Q_{j}$ for $j=1, \ldots, N$ and

$$
c_{k}=\frac{1}{k}\left(G_{k}-G_{k+1}\right) \quad \text { for all } k
$$

rewrite (8) as:

$$
\begin{array}{r}
\frac{d}{d t} G_{i}(t) \\
\leq \sum_{j=1}^{N} \sum_{k=i-j}^{i-1}(j+k)\left(a_{j k} c_{j} \frac{G_{k}-G_{k+1}}{k}-b_{j k} \frac{G_{j+k}-G_{j+k+1}}{j+k}\right) \\
+\sum_{j=1}^{N} \sum_{k=i-j}^{i-1} j a_{j, j+k} c_{j} \frac{1}{j+k}\left(G_{j+k}-G_{j+k+1}\right) \\
\leq \sum_{j=1}^{N} \sum_{k=i-j}^{i-1}(j+k)\left(a_{j k} z_{j} \frac{G_{k}-G_{k+1}}{k}-b_{j k} \frac{G_{j+k}-G_{j+k+1}}{j+k}\right) \\
+\sum_{j=1}^{N} \sum_{k=i-j}^{i-1} j a_{j, j+k} z_{j} \frac{1}{j+k}\left(G_{j+k}-G_{j+k+1}\right) \\
=\sum_{j=1}^{N} \sum_{k=i-j}^{i-1}\left(A_{j k}\left(G_{k}-G_{k+1}\right)-B_{j k}\left(G_{j+k}-G_{j+k+1}\right)\right)
\end{array}
$$

Where

$$
\begin{aligned}
A_{j k} & :=\frac{(j+k) a_{j k} z_{j}}{k} \\
B_{j k} & :=\frac{(j+k) b_{j k}-j a_{j, j+k} z_{j}}{j+k} .
\end{aligned}
$$

Now we take any $\lambda<\bar{\lambda}<\frac{z_{s}}{z}$ (recall $\lambda$ appears in the condition on $r_{i}$ ), and note that the following holds for $k$ large enough:

$$
B_{j k} \geq \bar{\lambda}^{j} A_{j k} \quad \text { for } j=1, \ldots, N .
$$

The proof of this is easy, as (note that we can divide by $a_{j k}$ by hypothesis 1):

$$
\begin{aligned}
\frac{B_{j k}}{A_{j k}}=\frac{k b_{j k}}{(j+k) a_{j k} z_{j}} & -\frac{j k a_{j, j+k} z_{j}}{(j+k)^{2} a_{j k} z_{j}} \\
= & \frac{k a_{j k} Q_{j} Q_{k}}{(j+k) a_{j k} Q_{j+k} Q_{j} z^{j}}-\frac{j k a_{j, j+k}}{(j+k)^{2} a_{j k}} \\
& =\frac{k}{j+k} \frac{Q_{k}}{Q_{j+k}} \frac{1}{z^{j}}-\frac{j k a_{j, j+k}}{(j+k)^{2} a_{j k}},
\end{aligned}
$$

where the detailed balance hypothesis 2 has been used to pass to the second line. Now observe that the term with the negative sign converges to 0 thanks to hypothesis [5] and that the other term

$$
\lim _{k \rightarrow \infty} \frac{k}{j+k} \frac{Q_{k}}{Q_{j+k}} \frac{1}{z^{j}}=\left(\frac{z_{s}}{z}\right)^{j}>\bar{\lambda}^{j}
$$


because of hypothesis 4 . Hence we have (10).

So, thanks to (10), we can continue from (9) and get, for $i$ large enough:

$$
\frac{d}{d t} G_{i} \leq \sum_{j=1}^{N} \sum_{k=i-j}^{i-1} A_{j k}\left(G_{k}-G_{k+1}-\bar{\lambda}^{j}\left(G_{j+k}-G_{j+k+1}\right)\right)
$$

It is easy to see from hypothesis 5 that for $j, m=1, \ldots, N$,

$$
\frac{A_{j k}}{A_{j, k+m}} \rightarrow 1 \quad \text { as } k \rightarrow \infty
$$

This means that for small variations of $k, A_{j k}$ changes little when $k$ is large. Take $\epsilon$ such that

$$
\frac{1-\epsilon}{1+\epsilon} \geq \frac{\lambda}{\bar{\lambda}}
$$

We can then find an $i_{0}>2 N$ such that (13) holds for $i \geq i_{0}$ and we have, also for $i \geq i_{0}$ :

$$
(1-\epsilon) A_{j, i-j} \leq A_{j k} \leq(1+\epsilon) A_{j, i-j} \quad \text { for } j=1, \ldots, N, \quad k=i-j, \ldots, i-1
$$

So for $i \geq i_{0}$ we can write from (13):

$$
\begin{array}{r}
\frac{d}{d t} G_{i} \\
\leq \sum_{j=1}^{N} A_{j, i-j} \sum_{k=i-j}^{i-1}\left((1+\epsilon)\left(G_{k}-G_{k+1}\right)-(1-\epsilon) \bar{\lambda}^{j}\left(G_{j+k}-G_{j+k+1}\right)\right) \\
=\sum_{j=1}^{N} A_{j, i-j}\left[(1+\epsilon)\left(G_{i-j}-G_{i}\right)-(1-\epsilon) \bar{\lambda}^{j}\left(G_{i}-G_{i+j}\right)\right]
\end{array}
$$

From the hypothesis on $r_{i}$, for $j=1, \ldots, N$ and $i>j$,

$$
\begin{aligned}
& r_{i-j}-r_{i}=\sum_{k=1}^{j}\left(r_{i-k}-r_{i-k+1}\right) \\
& \leq \sum_{k=1}^{j} \lambda\left(r_{i-k+1}-r_{i-k+2}\right)=\lambda\left(r_{i-j+1}-r_{i+1}\right)
\end{aligned}
$$

Apply this $j$ times to get:

$$
r_{i-j}-r_{i} \leq \lambda^{j}\left(r_{i}-r_{i+j}\right) \leq \bar{\lambda}^{j} \frac{1-\epsilon}{1+\epsilon}\left(r_{i}-r_{i+j}\right),
$$

where we used (14) together with $\lambda / \bar{\lambda}<1$ to say that

$$
\frac{1-\epsilon}{1+\epsilon} \geq\left(\frac{\lambda}{\bar{\lambda}}\right)^{j}
$$


If the sequence $\left\{r_{i}\right\}$ satisfies (17) then $\left\{C r_{i}\right\}$ also satisfies it, for any positive $C$. Take $C>1$ sufficiently large so that

$$
C r_{i}>M_{0} \geq G_{i}(t) \quad \text { for } i<i_{0} \text { and } t \leq T \text {, }
$$

where by $M_{0}$ we mean the density of the full initial data with no truncation. Now define

$$
\begin{aligned}
M_{i} & :=G_{i}-C r_{i}, \\
H_{i} & :=\left(G_{i}-C r_{i}\right)_{+}
\end{aligned}
$$

We know $H_{i}(t)=0$ for $i<i_{0}$ and $t<T$ because of (18).

As the $C r_{i}$ satisfy (17) we can write, continuing from (16), for $i \geq i_{0}$ :

$$
\frac{d}{d t} M_{i} \leq \sum_{j=1}^{N} A_{j, i-j}\left[(1+\epsilon)\left(M_{i-j}-M_{i}\right)-(1-\epsilon) \bar{\lambda}^{j}\left(M_{i}-M_{i+j}\right)\right]
$$

Then, the same inequality holds for $H_{i}$ : note that most of the previous reorganization was done in order to have the term in $M_{i}$ as the only term with negative sign in (21). Otherwise we cannot justify writing the inequality in terms of $H_{i}$ as is done next:

$$
\begin{aligned}
\frac{d}{d t} H_{i}=1_{M_{i}>0} \frac{d}{d t} M_{i} \leq \\
\leq 1_{M_{i}>0} \sum_{j=1}^{N} A_{j, i-j}\left[(1+\epsilon)\left(M_{i-j}-M_{i}\right)-(1-\epsilon) \bar{\lambda}^{j}\left(M_{i}-M_{i+j}\right)\right] \\
\leq \sum_{j=1}^{N} A_{j, i-j}\left[(1+\epsilon)\left(H_{i-j}-H_{i}\right)-(1-\epsilon) \bar{\lambda}^{j}\left(H_{i}-H_{i+j}\right)\right] .
\end{aligned}
$$

(We have used $1_{M_{i}>0} M_{i}=H_{i}$ and $1_{M_{i}>0} M_{k} \leq H_{k}$ for any $i, k$ ). Now we can sum this from $i=i_{0}$ to infinity (note again that the sums are all convergent, as the solution $\left\{c_{j}\right\}$ with truncated initial data has finite moments of all orders) 
and reorganize the terms:

$$
\begin{gathered}
\frac{d}{d t} \sum_{i=i_{0}}^{\infty} H_{i} \leq \sum_{i=i_{0}}^{\infty} \sum_{j=1}^{N} A_{j, i-j}\left[(1+\epsilon)\left(H_{i-j}-H_{i}\right)-(1-\epsilon) \bar{\lambda}^{j}\left(H_{i}-H_{i+j}\right)\right] \\
=\sum_{j=1}^{N} \sum_{i=i_{0}-j}^{\infty} H_{i}\left((1+\epsilon) A_{j i}-(1-\epsilon) \bar{\lambda}^{j} A_{j, i-j}\right)-\sum_{j=1}^{N} \sum_{i=i_{0}}^{\infty} H_{i}(1+\epsilon) A_{j, i-j} \\
+\sum_{j=1}^{N} \sum_{i=i_{0}+j}^{\infty} H_{i}(1-\epsilon) \bar{\lambda}^{j} A_{j, i-2 j} \\
=\sum_{j=1}^{N} \sum_{i=i_{0}-j}^{i_{0}-1} A_{j i}(1+\epsilon) H_{i}+\sum_{i=i_{0}}^{\infty} H_{i}(1+\epsilon) \sum_{j=1}^{N}\left[A_{j i}-A_{j, i-j}\right] \\
+\sum_{i=i_{0}}^{\infty} H_{i}(1-\epsilon) \sum_{j=1}^{N} \bar{\lambda}^{j}\left[A_{j, i-2 j}-A_{j, i-j}\right]-\sum_{j=1}^{N} \sum_{i=i_{0}}^{i_{0}+j-1} H_{i} A_{j, i-2 j} \bar{\lambda}^{j}(1-\epsilon)
\end{gathered}
$$

where the $T_{i}(\mathrm{i}=1,2,3,4)$ are the sums above. Observe that $T_{4}$ is negative and $T_{1}$ only contains terms in $H_{i}$ for $i<i_{0}$, so it is directly zero (recall (18)). Also, note that for $j=1, \ldots, N$ and $i \geq i_{0}$ we have, by using hypothesis [6] that

$$
\begin{aligned}
&\left|A_{j i}-A_{j, i-j}\right|=\left|\frac{j+i}{i} a_{j i} z_{j}-\frac{i}{i-j} a_{j, i-j} z_{j}\right| \\
& \leq z_{j} a_{j i}\left|\frac{j+i}{i}-\frac{i}{i-j}\right|+z_{j} \frac{i}{i-j}\left|a_{j i}-a_{j, i-j}\right| \\
& \quad \leq z_{j} K(i+j) \frac{j^{2}}{i(i-j)}+z_{j} \frac{i}{i-j} K_{a}
\end{aligned}
$$

which is easily seen to be bounded by a certain constant $A^{\prime}$ for $j=1, \ldots, N$ and $i>N$. Hence, the coefficient of $H_{i}$ in $T_{2}$ and $T_{3}$ is bounded by a certain constant $A$ independent of $j$ and $i$ and then

$$
\frac{d}{d t} \sum_{i=i_{0}}^{\infty} H_{i} \leq A \sum_{i=i_{0}}^{\infty} H_{i} .
$$

Gronwall's lemma then shows that $H_{i}(t)=0$ for $i \geq i_{0}$ and $t \in[0, T]$; that is to say $G_{i}(t) \leq C r_{i}$. This proves our claim.

\subsection{Proof of the main theorem}

Finally, we arrive at the proof of theorems 11 and 9] which is not difficult once the proposition of the previous section has been established.

Proof of Theorem 11. Let $c$ be an admissible solution that converges weak-* in $X$ to an equilibrium of mass $\rho<\rho_{s}$, which must be given by $\left\{Q_{j} \bar{z}^{j}\right\}_{j \geq 1}$ for 
some $0 \leq \bar{z}<z_{s}$ (see theorem 8). We will prove that the orbit of any such solution must be relatively compact in $X$ and hence the convergence must be strong.

Pick $z \in] \bar{z}, z_{s}\left[\right.$. As we know that $c_{j} \rightarrow Q_{j} \bar{z}^{j}$ when $t \rightarrow \infty$ for all $j$, we can find a $t_{0}>0$ so that

$$
c_{j}(t) \leq Q_{j} z^{j} \text { for all } j=1, \ldots, N \text { and } t \geq t_{0} .
$$

As Lemma 13 ensures, we can always find a sequence $\left\{r_{i}\right\}$ tending to zero as $i \rightarrow \infty$ that satisfies the conditions in Proposition 12 with $G_{i}\left(t_{0}\right)$ instead of $G_{i}(0)$. We can apply the proposition, with the $z$ we have chosen, to $\left\{c_{j}(t+\right.$ $\left.\left.t_{0}\right)\right\}_{j \geq 1}$ (which is a translation in time of the solution $c$ and thus is a solution itself) and deduce that for some $C>0, k_{0} \geq 1$ and all $t>t_{0}$,

$$
G_{i}(t) \leq C r_{i} \quad \text { for } i \geq k_{0} .
$$

As $\left\{r_{i}\right\}$ tends to zero, this bound says that the solution $c$ is relatively compact in $X_{+}$, and we have finished.

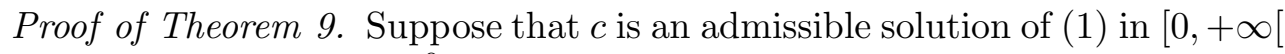
with initial data $c(0)=c^{0} \in X_{+}$. Theorem 10 shows that $c$ converges weak-* in $X$ to an equilibrium of mass $\rho$ for some $0 \leq \rho \leq \rho_{0}$.

If $\rho_{0}<\rho_{s}$, then this convergence is also strong (by theorem [11) and hence $\rho=\rho_{0}$.

If $\rho_{0}=\rho_{s}$ (with $\rho_{s}<\infty$ ), then $\rho \leq \rho_{s}$. But if $\rho<\rho_{s}$ then again the convergence must be strong and $\rho=\rho_{s}$, which is a contradiction. Hence, $\rho=\rho_{0}=\rho_{s}$ and we see the convergence is strong because of lemma 1 .

Finally, if $\rho_{0}>\rho_{s}$, then $\rho \leq \rho_{s}$ and it must be $\rho=\rho_{s}$ or otherwise the convergence is strong, which is not possible given that $\rho_{0}>\rho$.

\section{References}

[1] J. M. Ball, J. Carr, O. Penrose, The Becker-Döring cluster equations: basic properties and asymptotic behaviour of solutions, Comm. Math. Phys. 104, 657-692 (1986)

[2] J. M. Ball, J. Carr, Asymptotic behaviour of solutions to the Becker-Döring equations for arbitrary initial data, Proc. Roy. Soc. Edinburgh Sect. A, 108, 109-116 (1988)

[3] J. M. Ball, J. Carr, The discrete coagulation-fragmentation equations: existence, uniqueness and density conservation, J. Stat. Phys. 61, 203-234 (1990)

[4] R. Becker, W. Döring, Kinetische Behandlung der Keimbildung in übersättigten Dämpfen, Ann. Phys. (Leipzig) 24, 719-752 (1935)

[5] J. Carr, Asymptotic behaviour of solutions to the coagulationfragmentation equations. I. The strong fragmentation case, Proc. Roy. Soc. Edinburgh Sect. A, 121, 231-244 (1992) 
[6] J. Carr, F. P. da Costa, Asymptotic behavior of solutions to the coagulation-fragmentation equations. II. Weak fragmentation, J. Stat. Phys. 77, 89-123 (1994)

[7] F. P. da Costa, Asymptotic behaviour of low density solutions to the generalized Becker-Döring equations, NoDEA Nonlinear Differential Equations Appl. 5, 23-37, (1998)

[8] M. Escobedo, Ph. Laurençot, S. Mischler, B. Perthame, Gelation and mass conservation in coagulation-fragmentation models, J. Differential Equations, to appear.

[9] M. Escobedo, S. Mischler, B. Perthame, Gelation in coagulation and fragmentation models, Comm. Math. Phys. 231, 157-188 (2002)

[10] P.E. Jabin, B. Niethammer, On the rate of convergence to equilibrium in the Becker-Döring equations, J. Differential Equations 191, 518-543 (2003)

[11] Ph. Laurençot, On a class of continuous coagulation-fragmentation models, J. Differential Equations 167, 145-174 (2000)

[12] Ph. Laurençot, S. Mischler, Global existence for the discrete diffusive coagulation-fragmentation equation in $L^{1}$, Rev. Mat. Iberoamericana 18, 731-745 (2002)

[13] $\mathrm{Ph}$. Laurençot, S. Mischler, The continuous coagulation-fragmentation equations with diffusion, Arch. Rational Mech. Anal. 162, 45-99 (2002)

[14] $\mathrm{Ph}$. Laurençot, S. Mischler, On coalescence equations and related models, "Modelling and computational methods for kinetic equations", eds. P. Degond, L. Pareschi, G. Russo, in the Series Modelling and Simulation in Science, Engineering and Technology (MSSET), Birkhauser (2004)

[15] Ph. Laurençot, S. Mischler, Notes on the Becker-Döring equation, personal communication.

[16] Ph. Laurençot, S. Mischler, From the Becker-Döring to the LifshitzSlyozov-Wagner equations, J. Statist. Phys. 106, 5-6, pages 957-991 (2002).

[17] $\mathrm{Ph}$. Laurençot, The discrete coagulation equation with multiple fragmentation, Proc. Edinburgh Math. Soc. (2), 45, 67-82 (2002)

[18] S. Mischler, M. Rodríguez Ricard, Existence globale pour l'Equation de Smoluchowski continue non homogène et comportement asymptotique des solutions, C. R. Acad. Sci. Paris Sér. I Math 336, 407-412 (2003)

[19] O. Penrose, J. L. Lebowitz, Towards a rigorous theory of metastability, Studies in Statistical Mechanics, Vol. VII, eds. E. W. Montroll, J. L. Lebowitz, North-Holland (1979)

[20] M. Smoluchowski, Versuch einer mathematischen Theorie der Koagulationskinetik kolloider Lösungen, Zeitschrift für physik. Chemie 92, 129-168 (1917)

[21] I. W. Stewart, A global existence theorem for the general coagulationfragmentation equation with unbounded kernels, Math. Methods Appl. Sci. 11, 627-648 (1989) 
[22] I. W. Stewart, On the coagulation-fragmentation equation, Z. Angew. Math. Phys. 41, 917-924 (1990) 